Relations industrielles

Industrial Relations

\title{
Les lois Auroux
}

\section{Guy Caire}

Volume 39, numéro 2, 1984

URI : https://id.erudit.org/iderudit/050026ar

DOI : https://doi.org/10.7202/050026ar

Aller au sommaire du numéro

Éditeur(s)

Département des relations industrielles de l'Université Laval

ISSN

0034-379X (imprimé)

1703-8138 (numérique)

Découvrir la revue

Citer cet article

Caire, G. (1984). Les lois Auroux. Relations industrielles / Industrial Relations, 39(2), 235-258. https://doi.org/10.7202/050026ar
Résumé de l'article

L'auteur expose les grandes lignes des changements profonds apportes au Code du travail français par les lois Auroux en 1982. Il explicite les motivations qui éclairent leur présentation ainsi que l'accueil qui leur a été fait.
Tous droits réservés (C) Département des relations industrielles de l'Universite Laval, 1984
Ce document est protégé par la loi sur le droit d'auteur. L’utilisation des services d'Érudit (y compris la reproduction) est assujettie à sa politique d'utilisation que vous pouvez consulter en ligne.

https://apropos.erudit.org/fr/usagers/politique-dutilisation/ 


\title{
Les lois Auroux
}

\author{
Guy Caire
}

L'auteur expose les grandes lignes des changements profonds apportés au Code du travail français par les lois Auroux en 1982. Il explicite les motivations qui éclairent leur présentation ainsi que l'accueil qui leur a été fait.

«La révolution du bon sens! $\mathrm{j}$ 'aimerais assez que les nouveaux droits des travailleurs que je préconise portent ce label de qualité. Révolution, car j'espère créer les conditions d'un changement profond dans les relations du travail et susciter une rupture avec des pratiques périmées. Bon sens, car j'invite les hommes (et les structures également) à tirer meilleur parti d'euxmêmes: le travailleur et donc le cadre aussi peuvent en sortir grandis; le syndicat peut y trouver une présence et une autorité renforcées; l'entreprise, enrichie d'un nouvel investissement social peut en tirer une plus grande efficacité économique ${ }^{1}$.» Cette déclaration de J. Auroux ouvrant la première des livraisons de Droit social consacrées aux Réformes qui ont vu le jour depuis l'accession au pouvoir de la gauche situe bien, semble-t-il, l'esprit de ce qu'il est désormais convenu d'appeler les lois Auroux: une révolution paisible destinée à moderniser le système français de relations professionnelles, restaurant la dignité du travailleur, confortant la présence syndicale dans l'entreprise et libérant dans cette dernière des gains potentiels de productivité. Mais encore faut-il, pour situer pleinement le changement que cela implique, expliciter les motivations générales ou spécifiques qui éclairent les différents textes qui ont vu le jour, présenter les novations qui sont intervenues en explorant dans leurs grandes lignes les lois qui, en 1982, sont venues modifier 358 articles du Code du travail et enfin présenter l'accueil qui leur a été fait et les réactions qu'elles ont suscitées chez les partenaires sociaux concernés au premier chef. C'est cette démarche ternaire qui sera adoptée dans les développements qui suivent.

* CAIRE, Guy, professeur, Centre de recherche pour une nouvelle économie appliquée, Université de Parix X-Nanterre.

1 «La révolution du bon sens», Droit social, avril 1982, p. 258. 


\section{MOTIVATIONS}

Les rapports au Président de la République qui précèdent au journal officiel la publication des différents textes fournissent un éclairage des raisons de l'intervention du législateur dans chaque cas d'espèce. Mais si l'on veut rétablir la cohérence d'ensemble du dispositif ayant vu le jour en 1982, il convient d'adopter une perspective plus générale reposant sur trois séries de considérations complémentaires. Puisqu'il s'agit de «susciter une rupture avec des pratiques périmées», il convient tout d'abord de porter un jugement sur l'état des relations professionnelles en France en mai 1981. Puisqu'il s'agit aussi de réaliser «la révolution du bon sens» et même si comparaison n'est pas raison, il conviendra également d'apprécier, par différence et rapprochement avec des pays voisins de développement comparable, le champs des réformes possibles. Enfin, ce «changement profond dans les relations de travail» ressort d'un projet politique dont il importe de voir les fondements qui sont les siens et les options qu'il comporte.

\section{Constat français}

Absente du pouvoir depuis la cinquième République, la gauche a pu éprouver le besoin, bien légitime chez des héritiers, de dresser un état des lieux lors de sa prise de pouvoir. François Mitterand s'était d'ailleurs engagé, avant d'accéder à la charge suprême, à faire procéder à l'établissement d'un bilan détaillé et objectif de la situation de la France, principalement dans les domaines économique et social. La commission du bilan, présidée par $\mathrm{F}$. Bloch-Laine, va ainsi dresser un vaste panorama des forces et faiblesses françaises en mai 1981. Dans le domaine des relations professionnelles où le giscardisme et le barrisme avaient apporté un certain nombre d'éléments nouveaux, l'analyse se reconnaît d'emblée, «particulièrement inconfortable dans la mesure où, ici, toute généralité devient artificielle» ${ }^{2}$, d'une part parce que le tissu industriel est d'une très grande hétérogénéité et que, liée à la dimension variable des entreprises, la législation applicable aux communautés de travail est soumise à une série complexe de seuils ${ }^{3}$, d'autre part parce que, si la croissance avait permis des progrès (plus, il est vrai en matière de salaires que de conditions de travail), les incidences de la crise ont été différentes selon les secteurs et les entreprises. Il est néanmoins possible de faire un certain nombre d'observations.

2 «La France en mai 1981, forces et faiblesses», La Documentation française, 1982, p. 197.

3 C. SACKS, "Les seuils d'effectifs: une problématique en évolution?», Droit social, juillet-août 1983, pp. 472-485. 
Pour ce qui est des rapports de travail individuels les auteurs du rapport relèvent l'ampleur des inégalités salariales (en matière de salaires malgré une politique du SMIG qui a constitué l'instrument privilégié d'un relèvement des plus bas salaires et des efforts au plan des avantages sociaux complémentaires concernant notamment les catégories les plus défavorisées; en matière de durée de travail malgré un processus de réduction encouragé par des dispositions législatives; en matière aussi de conditions de travail où les pouvoirs réglementaire et disciplinaire de l'entrepreneur demeurent entiers, les travaux de la commission Rivero ayant été arrêtés, et où on constate une faillite à peu près complète de la répression des infractions relatives à l'hygiène et à la sécurité); le rapport constate aussi l'échec des politiques de participation: les travaux, et les recommandations de la commission Sudreau sont restés lettre morte pour l'essentiel; seule l'institution du bilan social en 1977 peut être considérée comme un acquis positif; enfin, le rapport constate l'essor des stratégies patronales de précarisation et extériorisation des emplois avec les pratiques de travail intérimaire (pour lequel les suggestions du rapport Couste de 1979 sont demeurées sans suite), sous-traitance, contrats à durée déterminée etc. qui permettent d'échapper aux dispositions sur le licenciement, à celles des conventions collectives, aux lois sur la représentation du personnel, qui brisent la communauté de travail et font obstacle à une action efficace.

Ces stratégies ne sont pas à leur tour sans influencer les relations collectives de travail en mettant à mal les institutions représentatives avec l'éclatement des statuts des personnels et l'éclatement de la notion même d'entreprise $^{4}$ - ce à quoi tentent malaisément de répondre quelques rares arrêts de la Cour de Cassation ou du Conseil d'État en forgeant le concept de groupe ou celui d'unité économique et sociale - . Par ailleurs, le système de négociation se révèle complexe (le niveau de la négociation dépendant entièrement des structures et des attitudes des organisations patronales) et inefficace (une part importante des salariés restant dépourvue de toute couverture conventionnelle) et le droit de grève demeure ambigu.

Ces différents thèmes que développe le rapport annexe préparé par $P$. Saint-Jevin débouchent sur trois considérations principales:

"- la relation de travail s'est trouvée profondément modifiée avec l'avènement de nouvelles politiques de personnel et sous la pression de la crise de l'emploi...

- d'importants «points noirs» subsistent dans la condition de certaines catégories de salariés qui cumulent fréquemment les caractéristiques les plus défavorables... 
- enfin l'absence de toute réforme d'ensemble du système des relations de travail, pourtant nécessaire, est frappante»s 5

\section{Comparaisons internationales}

Sans doute tout système de relations professionnelles a-t-il une spécificité qui interdit d'effectuer une transposition pure et simple des dispositions qui le particularisent. Dans la mesure cependant où les nouveaux droits accordés aux travailleurs français par les lois Auroux et qui peuvent paraître exhorbitants aux yeux de certaines fractions du patronat existent déjà pour nombre d'entre eux à l'étranger et vont même parfois au delà des dispositions françaises, il peut être utile d'en tenter un rapide survol, d'autant que partout, quoique sous des modalités différentes, tente de se faire jour une démocratie économique qu'on peut caractériser par trois traits essentiels: «Le respect des droits de l'homme sur les lieux de travail; la consolidation de la liberté sociale et politique; l'établissement d'un nouveau régime de relations professionnelles» ${ }^{6}$. Or, ce sont bien là les visées de la loi du 4 août 1982 sur les droits des salariés, de la loi du 28 octobre 1982 sur les institutions représentatives dans l'entreprise et de la loi du 13 novembre 1982 sur la politique contractuelle.

Pour ce qui concerne le premier thème, en Italie, selon le statut des travailleurs du 10 mai 1970 qui s'applique à toutes les entreprises de plus de quinze salariés et dont l'idée maîtresse est de faire des travailleurs de véritables sujets politiques, les travailleurs ont le droit de manifester leur pensée sur le lieu de travail et de se réunir en assemblée pendant la durée du travail. Au Danemark, les problèmes d'hygiène et de sécurité sont, depuis le ler octobre 1978, de la compétence d'un délégué spécialisé; en Suède, les lois de 1974 et de 1978 donnent la possibilité au délégué à la sécurité et même au simple employé d'interrompre immédiatement la production s'il estime que la tâche accomplie comporte des risques physiques. En RFA, les syndicats peuvent s'opposer à des licenciements et exiger un «plan social» pour atténuer les conséquences des mesures envisagées par la direction.

Concernant le second thème, en Italie, le conseil d'entreprise, seul contre-pouvoir, cumule les fonctions de la section syndicale, du délégué du personnel, du comité d'entreprise; il peut, en cas de conflit, faire appel à un expert extérieur. En RFA, le comité d'entreprise a un rôle prépondérant dans toutes les questions sociales et de personnels. Au Danemark, depuis

5 "La France en mai 1981, III. La vie collective et les politiques sociales", la Documentation française, 1982, p. 233. p. 148.

6 C. LEVINSON, Le contre-pouvoir multinational, la riposte syndicale, Le Seuil, 1974, 
1976, les employeurs contribuent au financement de la formation des délégués du personnel et, depuis 1974, le personnel des groupes de holdings a le droit d'élire deux représentants au conseil d'administration. En Suède, toute une panoplie de lois (sur la co-détermination, l'environnement du travail, la protection de l'emploi, la représentation du personnel dans les conseils d'administration) imposent à l'employeur l'obligation d'informer les représentants du personnel sur la vie de l'entreprise et de négocier toutes les décisions qui impliquent modification de la situation d'un ou de plusieurs employés.

Concernant le troisième thème en Italie, les conquêtes successives ont été sanctionnées par des conventions collectives plus que par la législation; le conseil d'usine ou les comités qui en dépendent négocient aussi bien les salaires que les cadences, l'organisation du travail, l'hygiène ou la sécurité; dès 1978, la convention collective consacrait un droit de regard syndical sur l'investissement et la politique de développement. En RFA, si les salaires sont régis par des conventions collectives de branches, les comités d'entreprise interviennent par la mise au point des primes et des bonifications.

On aurait pu multiplier les exemples ${ }^{7}$ mais les quelques illustrations cidessus suffisent à montrer que les dispositifs mis en place par les lois Auroux sont loin d'avoir le caractère radical que d'aucuns leur prêtent. Les libertés individuelles dans l'entreprise, l'information et la consultation des institutions représentatives, le champ concédé à la négociation sont sans doute plus larges ailleurs qu'ils ne le sont en France depuis les mutations de 1982 qui s'inscrivent dans les limites d'un compromis social définies par l'histoire et les pratiques sociales de notre pays.

\section{Projets politiques}

La campagne présidentielle de 1981 a été l'occasion pour chacun des candidats de présenter leur programme, programmes dans lesquels les questions professionnelles avaient l'importance qu'on peut deviner ${ }^{8}$. Le pouvoir en place ne manquait pas d'idées: Valéry Giscard d'Estaing avait imposé le bilan social, la loi sur la concertation avec les cadres; Beullac. Dans une lettre au Conseil national du patronat français (CNPF), avait rappelé l'importance et l'urgence d'une mise en place du droit d'expression directe; Stoleru préparait le projet Propage créant l'expression des salariés avec un budget mis à la disposition des ateliers pour être discuté et utilisé. Du côté de ce qui était à l'époque l'opposition on n'était pas en reste.

7 On peut par exemple consulter, European Industrial Relations by Industrial Democracy in Europe (IDE), International Research Group, Clarendon press 1981.

8 Le Monde, dossiers et documents, supplément, mai 1981. 
La gauche proclamait en effet sa volonté d'entreprendre «une réforme profonde de la législation du travail»", fondée sur deux idées essentielles que développe le premier chapitre de la seconde partie du «programme commun de gouvernement», texte ratifié le 27 juin 1972. D'une part, l'intervention des travailleurs et de leurs organisations dans l'entreprise devait reposer sur des conventions collectives et des accords d'entreprise comportant obligatoirement des dispositions concernant les cadences, l'aménagement des horaires de travail, le temps de congé et l'affectation aux postes de travail, sur une consultation obligatoire des comités d'entreprise et d'établissement et des délégués du personnel avant toute mesure concernant l'embauche, le licenciement, l'affectation aux postes de travail, les mutations, la classification des travailleurs, la détermination des cadences et plus généralement l'ensemble des conditions de travail et enfin sur l'élargissement des moyens matériels, financiers et de personnel des institutions représentatives (C.E. délégués du personnel, sections syndicales). D'autre part, la gestion démocratique du secteur public et nationalisé devait, quant à elle, être assurée par une réhabilitation du tripartisme instauré en 1945, renforcée par l'extension des pouvoirs des travailleurs en ce qui concerne les problèmes du personnel mais aussi les objectifs de production et d'investissement.

De son côté, le Manifeste socialiste adopté au Congrès de Créteil le 24 janvier 1981 proclamait que: le contrat à durée indéterminée redeviendra la base des relations de travail, les capacités d'intervention du syndicat dans l'entreprise seront étendues et affermies: moyens et protections des délégués élus, temps consacré à l'information et à l'expression collective (point 22). Par ailleurs, faisant écho aux revendications ouvrières, dont certaines trouveront satisfaction dans les lois Auroux, le Manifeste recensait toute une série de mesures susceptibles d'être adoptées: pouvoir du comité d'hygiène et de sécurité (CHS) d'arrêter les machines pour raison de sécurité; information du comité d'entreprise et droit de veto pour l'embauche, le licenciement, l'organisation du travail, le plan de formation, les nouvelles technologies (point 60); décentralisation du secteur public (point 62); participation des cadres au sein des organes représentatifs (point 63) etc.

\section{DISPOSITIONS}

Un temps pour la réflexion, un temps pour l'action. Il ne pouvait en effet être question, une fois la gauche venue au pouvoir, d'une valsehésitation. D'un ensemble de revendications, peut-être quelque peu disparates, il convenait de faire un tout cohérent. C'est à quoi s'est employé le

9 Programme commun de gouvernement, Éditions sociales, 1972, p. 58. 
nouveau ministre du travail, d'abord en dégageant une philosophie de l'action, ensuite en veillant à en assurer l'application dans des dispositifs juridiques précis et détaillés.

\section{Idées - forces}

Le rapport Auroux de septembre 1981 repose sur deux idées-forces et préconise quatre séries de mesures. En effet, «citoyens dans la cité, les travailleurs doivent l'être aussi dans l'entreprise»; il faut pour cela «ouvrir de nouveaux espaces de liberté et de responsabilité», les droits nouveaux qui en résulteront devant, à leur tour, «permettre de libérer les forces créatrices de l'entreprise» car «les travailleurs dans l'entreprise constituent un potentiel souvent mal utilisé de compétences, d'innovations et de talents; il s'agit là d'un gisement précieux non encore mis en valeur» ${ }^{10}$.

Première idée directrice: les travailleurs doivent être citoyens à part entière dans l'entreprise, ce qui va justifier deux séries de mesures. Il s'agit en premier lieu de restaurer et d'élargir les droits des salariés. Ce qui doit résulter de la réaffirmation d'un certain nombre de droits fondamentaux (droit au travail, à la formation, à la santé et à la sécurité dans l'entreprise, à l'information) mais aussi de la création de droits nouveaux: droit à l'exercice des libertés publiques dans l'entreprise par limitation et contrôle judiciaire du pouvoir disciplinaire du chef d'entreprise, par modification du règlement intérieur dans son contenu, ses procédures d'élaboration, son contrôle de légalité; droit d'expression des travailleurs sur leurs conditions de travail qui "«doit être direct, porter sur les conditions de travail, s'organiser autour du groupe ou de la cellule de travail, mettre en oeuvre les compétences de l'encadrement et prévoir l'articulation nécessaire avec les fonctions et les responsabilités des institutions représentatives et le nécessaire contrôle syndical sur la définition des procédures». Si les conditions de travail sont le terrain privilégié de l'expression des travailleurs dans l'entreprise, ceci requiert à son tour un renforcement des institutions représentatives existantes spécialisées dans le traitement de ces questions, ce qui sera obtenu par fusion du CHS et de la commission pour l'amélioration des conditions de travail (CACT) en une institution unique, généralisée, aux attributions étendues, ayant un véritable statut d'institution représentative et dotée de moyens adaptés (crédits d'heures et formation de ses membres); nécessite peut-être un aménagement de la médecine du travail déjà partiellement réalisé par le décret du 20 mars 1979; implique enfin que l'expression sur les conditions de travail (qui doit porter sur les conditions de travail, être

10 Nous citerons abondarnment dans les paragraphes qui suivent le texte de J. Auroux, «Les droits des travailleurs», rapport au Président de la République et au Premier Ministre, $L a$ Documentation française, 1981. 
directe, être celle du groupe de travail, être organisée contractuellement) puisse être articulée avec la fonction de médiation remplie, en ce domaine, par les institutions représentatives. Il s'agit en second lieu de reconstituer la collectivité de travail. En effet, depuis la crise «les entreprises ont développé de nouvelles formes de gestion de la main-d'oeuvre fondée sur la réduction des coûts et la recherche de la flexibilité: réduction à court terme des effectifs et organisation à moyen terme de la flexibilité de la main-d'oeuvre qu'elles utilisent. Les principales caractéristiques de ces nouvelles formes de gestion consistent à réduire à un "noyau dur» une partie des travailleurs titulaires d'un statut de protégé et à multiplier les formes de travail précaires». S'il convient pour lutter contre cette précarisation de revoir les statuts des travailleurs à temps partiel et à domicile, de lancer une réflexion sur la sous-traitance et le télétravail, c'est surtout la législation sur le travail temporaire et le contrat à durée déterminée qu'il convient de modifier. Pour cela, il convient de définir un cadre limité et précis pour les modalités de recours à ces formules en précisant clairement et limitativement pour quels motifs il peut y être fait appel, en en interdisant l'utilisation pour de longues durées, des durées incertaines ou pour tenir des postes de travail permanents; il convient aussi d'assurer à l'ensemble des salariés un traitement similaire à celui des salariés permanents en limitant la spécificité de leur statut aux seules nécessités reconnues.

Deuxième idée directrice: les travailleurs doivent devenir les acteurs du changement dans l'entreprise en pouvant peser et influer sur les décisions qui les intéressent directement. Pour cela deux types d'actions s'avèrent nécessaires. Il faut tout d'abord donner aux institutions représentatives un rôle accru. Ceci résultera tout à la fois d'un renforcement de la représentation des travailleurs en réformant les conditions de mise en place des institutions là où la loi les prévoit, en incitant à leur mise en place là où elle ne les prévoit pas, en permettant la représentation de chaque catégorie de travailleurs et en dotant les institutions représentatives de moyens supplémentaires (en effectifs, crédits d'heures, formation, possibilités d'expertise). D'une façon plus précise les comités d'entreprise devraient avoir un rôle accru, d'une part parce que le champ d'application de la législation les concernant serait élargi aux établissements publics industriels et commerciaux, aux groupes, et d'autre part parce que leur fonction économique serait revalorisée grâce à une information mieux adaptée, qu'il s'agisse d'une information économique ou sociale ou d'une information technologique, notamment dans le contexte de l'informatisation des entreprises, grâce a la mise en place d'une délégation économique dans les entreprises de plus de 1000 salariés et enfin en conférant au comité d'entreprise un droit d'alerte au cas de difficultés économiques de l'entreprise; de plus les comités d'en- 
treprise pourraient disposer désormais d'un budget de fonctionnement indépendant du budget des oeuvres sociales. Une seconde modalité devant permettre aux travailleurs de devenir des acteurs actifs du changement passe par la relance d'une politique contractuelle active; pour cela il faut remédier au «vide conventionnel» et faire en sorte que chaque salarié soit couvert par une convention collective et faire du droit des conventions collectives un stimulant de la négociation (en accroissant la fréquence de conclusion des conventions collectives, en étendant leur contenu, en renforçant leur crédibilité, en en assurant une meilleure diffusion auprès des travailleurs, en en garantissant l'application). Dans cette perspective, le Ministère du travail doit pouvoir jouer un rôle incitatif et offrir les moyens du développement de la négociation collective; mais surtout «la novation majeure dans la mise en oeuvre des droits nouveaux pour les travailleurs réside en l'instauration d'une obligation de négociation annuelle entre l'employeur et les organisations représentées dans l'entreprise», négociation portant prioritairement sur les salaires, la durée et les conditions de travail.

Au total, le cadre nouveau tracé au droit du travail s'efforce de «concilier deux impératifs qui, plus que jamais, sont liés, le premier de ces impératifs est de mettre en oeuvre des forces de progrès et de clarté qui permettent aux travailleurs d'accéder dans la société à la place qui doit être la leur et d'agir sur leur vie quotidienne, directement ou par la négociation des conditions de leur travail dans les entreprises, plutôt que de céder à la facilité en développant des mécanismes qui favorisent des antagonismes et exacerbent des blocages. Le second est de faire coexister la reconnaissance des droits nouveaux aux travailleurs et les divers équilibres de l'entreprise en libérant la capacité d'innovation et d'adaptation des travailleurs, indispensable dans les circonstances économiques difficiles que nous connaissons». On voit ainsi que la philosophie qui inspire le rapport Auroux s'inscrit dans la recherche d'un nouveau compromis social.

\section{Mesures}

Le rapport Auroux qui au plan de la procédure, en appelait à quelques grands principes: libérer les forces créatrices de l'entreprise, éviter les blocages législatifs, relancer l'esprit d'initiative, a été rendu public le 8 septembre 1981; il a été adopté par le Conseil des ministres le 4 novembre 1981; sa traduction juridique s'est faite par deux ordonnances et cinq projets de loi soumis pour avis au conseil économique et social les 9 et 10 mars 1982. Il est possible de regrouper ces textes autour de quatre axes essentiels, ceux mêmes énoncés par le rapport Auroux. 
Le premier axe des réformes est celui de la restauration et de l'élargissement des droits des salariés. À partir du constat que, jusque là, n'existait aucune limite claire au pouvoir disciplinaire du chef d'entreprise et que le principal instrument de ce pouvoir était le règlement intérieur qui organise de façon unilatérale les conditions de vie et la discipline dans l'entreprise, les pouvoirs publics vont élaborer un premier projet de loi - qui deviendra la loi $n^{\circ} 82.689$ du 4 août 1982 - réformant le règlement intérieur et définissant les grandes lignes d'un droit disciplinaire ${ }^{11}$. Il faut en effet noter qu'en de nombreux pays le règlement intérieur n'est plus la création du seul chef d'entreprise, que déjà, sous le précédent septennat, une commission présidée par le Professeur Rivero avait relevé la gravité et l'illégalité de certaines pratiques et formulé des propositions de réforme. Aussi, comme l'observe l'exposé des motifs «absence de détermination légale des matières relevant du règlement intérieur et son caractère essentiellement répressif ont conduit à des critiques de plus en plus sévères allant jusqu'à une remise en cause de l'institution elle-même. La nécessité de tenir compte de l'évolution des rapports sociaux dans le sens d'un transfert de certains domaines relevant traditionnellement du règlement intérieur à la négociation collective jointe à l'unanime aspiration des salariés au strict respect des libertés publiques dans l'entreprise ont rendu indispensable une réforme de la législation relative au règlement intérieur ainsi que l'intervention d'un véritable droit disciplinaire». Désormais, le règlement intérieur qui conserve son caractère unilatéral doit être un document écrit; obligatoire dans les entreprises de 20 salariés il ne doit contenir que des mesures relatives à la discipline, à l'hygiène et à la sécurité; soumis pour avis aux représentants du personnel, son contrôle appartient à l'inspection du travail de même que les notes de service portant prescriptions générales et permanentes qui peuvent lui être assimilées. Sont de même définies les grandes lignes d'un pouvoir disciplinaire: la sanction disciplinaire est définie; la nature et l'échelle des sanctions applicables doivent être énoncées; le salarié doit être informé par écrit, bénéfice d'un droit de défense; il existe une prescription de trois mois pour les faits et de trois ans pour la sanction; les prud'hommes contrôlent l'adéquation de la sanction à la faute commise. Le droit d'expression aurait pu faire l'objet d'un texte de loi distinct de celui du droit disciplinaire; il n'en a rien été et c'est le même texte qui concerne les deux domaines. En ce domaine, la loi cadre

11 Différents colloques ont abordé récemment cette question: Droit social, octobre 1981, Association française de droit du travail, novembre 1981, Droit ouvrier, mai 1982, Syndicat des avocats de France, décembre 1982; par ailleurs différentes études ont été consacrées à ce thème: G. BELLIER, «Droit disciplinaire et citoyenneté dans l'entreprise dans la réforme des droits des travailleurs", Droit Social, mai 1982, pp. 407-417; F. VENIN, «L'aménagement du pouvoir disciplinaire de l'employeur", Droit social, juillet-août 1983, pp. 486-493 et surtout le numéro spécial de Droit social de septembre-octobre 1982 tout entier consacré à la loi du 4 août 1982 relative aux libertés des travailleurs dans l'entreprise. 
proposée, si elle reconnaît au salarié le droit de s'exprimer sur ses conditions de travail, sur les lieux de travail et le temps d'expression étant payé, laisse aux partenaires le soin d'en arrêter les modalités concrètes, des négociations devant être engagées dans un délai de six mois dans les entreprises de plus de 200 salariés et le comité d'entreprise établissant un rapport d'évaluation des résultats, dans un délai de deux ans ${ }^{12}$. Au 15 mai 1983, 1043 accords témoignant d'une grande diversité dans leur dispositif et leur rédaction, représentant $12,1 \%$ des entreprises concernées avaient pu être recensés par les directions régionales du travail et de l'emploi ${ }^{13}$.

Le second axe des réformes est celui de la reconstitution de la collectivité de travail que la multiplication des statuts (travail intérimaire, contrats à durée déterminée, travail saisonnier, etc.) a fait éclater. Trois grands principes, clairement affirmés dans les rapports au Président de la République accompagnant les textes des ordonnances vont s'appliquer au travail temporaire (ordonnance du 5 février 1982), au contrat à durée déterminée (ordonnance du 5 février 1982), et au travail à temps partiel (ordonnance du 26 mars 1982); il convient de préciser clairement pour quels motifs il peut être fait appel à ces formules dérogatoires du droit commun afin d'en limiter le recours à des cas précis justifiés économiquement; il faut en limiter l'utilisation pour des emplois à caractère permanent en incitant les entreprises à adopter une meilleure gestion de leur personnel et en rendant le recours à ces formules dérogatoires du droit commun moins attractif pour les employeurs en en augmentant le coût; il faut enfin calquer le plus possible le statut des salariés concernés sur celui des salariés permanents à contrat à durée indéterminée, en améliorant les droits et protections de ces travailleurs ${ }^{14}$.

Le troisième axe des réformes est celui du rôle accru dévolu aux institutions représentatives. Cinq institutions sont visées par la loi $n^{\circ} 82.915 \mathrm{du} 28$ octobre 1982 dont la philosophie générale, clairement présentée dans l'exposé des motifs est que «le développement d'une véritable démocratie économique suppose que des relations sociales nouvelles s'établissent dans la collectivité de travail que constitue l'entreprise. Ainsi une meilleure combinaison des données économiques et des impératifs sociaux et humains sera

12 N. CATALA, «Les modalités d'expression des salariés dans l'entreprise», Droit social, septembre-octobre 1983, pp. 557-560.

13 B. BOYER, «Bilan de l'application de la loi sur l'expression des salariés au 15 mai 1983», Droit social, juillet-août 1983, pp. 445-450.

14 G. POULAIN, "La réforme du contrat de travail à durée déterminée», Droit social, avril 1982, pp. 356-371; Y. CHALARON, «La réforme du travail temporaire», Droit social, avril 1982, pp. 372-388; B. TEYSSIE, «Le travail à temps partiel», Droit social, mai 1982, pp. 396-406; A. LYON-CAEN, «Le recours au travail à durée limitée», Droit social, janvier 1983, pp. 5-16; J. PELISSIER, «Travail à durée limitée et droits des salariés», Droit social, janvier 1983, pp. 17-25. 
assurée. Pour atteindre ce but, il est nécessaire de renforcer et d'élargir les capacités des institutions représentatives du personnel et de les doter de pouvoirs leur permettant d'influer sur les décisions qui concernent directement les salariés»; concernant les syndicats, leur présence dans l'entreprise est renforcée par des structures mieux adaptées aux réalités de l'entreprise grâce à la constitution possible d'une section syndicale dans toutes les entreprises quelle que soit leur taille et par la désignation d'un délégué syndical central dans les plus grandes: on a donc une généralisation du droit syndical à la fois vers le haut et vers le bas avec la suppression de deux seuils d'effectifs (150 salariés pour l'attribution d'heures de délégation, 50 salariés pour la constitution de sections syndicales); elle l'est aussi par des moyens accrus donnés aux sections d'entreprise (collecte des cotisations possible pendant le temps de travail, libre contenu de l'information syndicale, local propre à chaque section dans les plus grandes entreprises); elle l'est enfin grâce à une solidité plus grande du mandat de délégué syndical que ce soit au plan de la désignation, de la protection ou de l'exercice des fonctions (libre circulation à l'intérieur de l'entreprise, crédit d'heures augmenté). Mais, si le syndicalisme voit son champ d'action élargi dans le cadre d'un pluralisme partiellement affiné, la liberté syndicale demeure encore largement formelle et surveillée, contrôlée qu'elle demeure par l'employeur ${ }^{15}$. Concernant les délégués du personnel, l'innovation la plus spectaculaire et qui a donné lieu à de longs débats devant l'Assemblée Nationale est celle du délégué de site devant permettre aux petites entreprises d'une zone industrielle, d'une galerie marchande, voire d'une rue ou d'un quartier de bénéficier d'une représentation qui leur manquait jusqu'à présent. Par ailleurs, dans les entreprises d'au moins $\mathbf{5 0}$ personnes qui n'ont pas de comité d'entreprise alors que légalement elles devraient en être dotées, les délégués du personnel bénéficieront d'une délégation des compétences dont le comité est normalement investi avec les moyens correspondants (information, experts et crédits d'heures particulier). Enfin, les délégués du personnel dont le nombre a été modifé par le décret $n^{\circ} 83.470$ du 8 juin 1983 pris en application de la loi, exerceront à l'avenir leurs fonctions dans de meilleures conditions: le droit de libre circulation est reconnu, le temps de réunion avec l'employeur est payé comme temps de travail sans imputation sur le crédit d'heures, les heures de délégation sont réputées de plein droit être «bien utilisées» conformément à leur objectif. Pour ce qui est des comités d'entreprise, la loi nouvelle commence par donner une définition du rôle qui leur est imparti, à savoir «assurer une expression collective des salariés, permettant la prise en compte permanente de leurs intérêts, dans les décisions relatives à la gestion et à

15 J.M. VERDIER, «Les réformes et le droit syndical», Droit social, 1982, pp. 291-298; "La présence syndicale dans l'entreprise et la loi du 28 octobre 1982 relative au développement des institutions représentatives du personnel», Droit social, janvier 1983, pp. 37-54. 
l'évolution économique et financière de l'entreprise, à l'organisation du travail et aux techniques de production». Pour assurer ses missions le comité d'entreprise est doté d'une panoplie de moyens qu'il ne possédait pas jusqu'alors (information accrue, possibilité de recourir à plusieurs types d'experts, formation économique de ses membres, instauration d'une commission économique dans les grandes entreprises etc.). Dans l'ordre économique et financier le comité d'entreprise doit essentiellement être informé et consulté. Certaines informations et consultations sont obligatoires: celles concernant la marche générale de l'entreprise, celles relatives aux modifications dans l'organisation de l'entreprise, celles qui concernent l'introduction de nouvelles technologies, celles qui concernent la politique de recherche de l'entreprise; d'autres sont facultatives comme par exemple pour ce qui est des augmentations de prix. Dans le domaine socio-économique, les attributions sont essentiellement consultatives: il en est ainsi en matière d'emploi, de conditions de travail, de travail précaire. La loi nouvelle apporte cependant quelques innovations: le comité d'entreprise peut organiser des réunions internes au personnel, peut inviter des personnalités extérieures; il dispose désormais d'une subvention de fonctionnement égale à $0,2 \%$ de la masse salariale brute ${ }^{16}$. La loi du 28 octobre 1982 comporte une innovation majeure: l'instauration de comités de groupe. L'exposé des motifs de la loi est à cet égard très claire sur les objectifs recherchés et les finalités attendues de l'institution qui, pour la délimitation du groupe, fait référence à la législation commerciale, laquelle définit les filiales et les participations: «la création de cette institution permettra aux représentants des comités d'entreprise des diverses entreprises du groupe de recevoir des informations de l'autorité responsable de la stratégie du groupe. Les informations reçues par les comités d'entreprise des sociétés filiales ne sont que parcellaires puisque le centre de décision est extérieur à l'entreprise; la constitution du comité de groupe assure aux représentants du personnel une information beaucoup plus complète, au niveau de l'unité économique d'ensemble constituée par le groupe... Le comité du groupe recueillera une information sur la situation économique du groupe, sur l'évolution de l'emploi au sein de celui-ci et sur les relations financières internes, il examinera notamment les comptes et bilans consolidés établis au niveau du groupe avec, s'il le souhaite, l'assistance d'un expert comptable». Le choix du législateur et les modalités adoptées procèdent donc, on le voit, de l'idée que les problèmes doivent être traités au plus près des niveaux où ils se

16 A. LYON-CAEN, «Le comité d'entreprise à l'heure du changement», Droit social, avril 1982, pp. 299-304; G. COUTURIER, «L'accès du comité d'entreprise à l'information économique et financière», Droit social, janvier 1983, pp. 26-36; A. COHEN, «Les nouvelles fonctions économiques du comité d'entreprise», Droit social, mars 1983, pp. 165-174 et surtout le numéro spécial de Droit social, juin 1983, «Le nouveau droit des comités d'entreprise». 
posent $^{17}$. La dernière institution représentative du personnel dans l'entreprise a fait, quant à elle, l'objet d'un texte spécifique, la loi $\mathrm{n}^{\circ} 82.1097 \mathrm{du}$ 23 décembre 1982. Cette loi a pour objectif de fournir un cadre privilégié pour la prévention des risques du travail et l'amélioration des conditions de travail, aussi fusionne-t-elle en un seul organisme le CHS et la commission pour l'amélioration des conditions de travail. Une autre nouveauté de la loi est la procédure d'intervention en cas de danger immédiat qui implique, dans cette éventualité, réunion du comité d'hygiène de sécurité et des conditions de travail (CHSCT) et, à défaut d'accord, saisie immédiate de l'inspecteur du travail. Cette disposition est quelque peu en retrait par rapport à celle qu'on trouvait dans les $\mathbf{1 1 0}$ propositions du programme Mitterand, qui prévoyait la possibilité d'arrêter les machines mais qui aurait risqué de poser le problème de la responsabilité civile voire pénale du travailleur et qui aurait été en contradiction avec le principe d'unité du pouvoir de décision. Par ailleurs, devant émettre un avis sur les points pour lesquels il est consulté le CHSCT devient une institution représentative, bénéficiant d'un crédit d'heures global, ayant la possibilité de faire appel à des experts rémunérés par l'entreprise et bénéficiant d'un droit à la formation ${ }^{18}$.

Le quatrième et dernier axe de la politique Auroux est celui de la relance de la politique contractuelle. Quatre défauts caractérisaient la situation antérieure: l'existence de branches ou d'entreprises non couvertes par une convention collective; la faible crédibilité des conventions signées par des organisations peu représentatives; le caractère irréel de certains accords, par exemple en matière de salaires; l'absence de négociation dans trop d'entreprises sur des sujets intéressant directement les travailleurs. La loi $\mathrm{n}^{\circ}$ 82.957 du 13 novembre 1982 relative à la négociation collective et au règlement des conflits collectifs de travail a pour objectif «de faire en sorte que la politique contractuelle devienne la pratique privilégiée du progrès social dans notre pays». Cette loi comporte une série de dispositions qu'on peut considérer comme secondaires et trois innovations essentielles. Rentrent, à notre avis, dans la première catégorie, la mise en place de commissions paritaires dans les entreprises de moins de onze salariés, les procédures d'extension (avec de nouvelles clauses obligatoires, extension autoritaire au cas de carence à des branches proches présentant des conditions économiques analogues), le recours à la médiation comme procédure de règlement des conflits, alors même que ces procédures de règlement pacifique (conciliation officielle, médiation, arbitrage) sont - comme le reconnaissent tous les spécialistes - de véritables branches mortes du droit. Rentrent dans la seconde

17 G. BELIER, «Mise en place et attributions du comité de groupe: de l'unité économique et sociale à la directive Vredeling", Droit social, juillet-août 1983, pp. 439-444.

18 P. CHAUMETTE, "Le comité d'hygiène, de sécurité et des conditions de travail et le droit de retrait du salarié», Droit social, juin 1983, pp. 425-433. 
catégorie l'obligation pour tous les organismes liés par un accord de branche de se réunir pour négocier sur les salaires une fois par an et pour réexaminer les classifications une fois tous les cinq ans; l'obligation pour le chef d'entreprise d'engager chaque année une négociation avec les organisations syndicales représentatives sur les salaires; le temps et l'aménagement du temps de travail, le droit de veto accordé aux organisations syndicales ayant obtenu plus de $50 \%$ des voix aux dernières élections du comité d'entreprise pour l'application dans l'entreprise d'accords dérogatoires au droit commun ${ }^{19}$.

Au total, les nouveaux textes qui contiennent nombre de mises à jour, d'incorporations à la loi de solutions jurisprudentielles, d'adaptations rédactionnelles ne comportent en fait que deux nouveautés essentielles: l'obligation de négocier le droit d'expression des salariés dans l'entreprise. Les partenaires sociaux ne s'y sont pas trompés puisque c'est dans ce domaine, ainsi que nous allons le voir, que les réactions ont été les plus vives.

\section{RÉACTIONS}

Les débats auxquels ont donné lieu au Conseil économique et social les projets Auroux ont donné une claire indication sur les lignes de partage qui devaient s'établir et qui fracturent encore actuellement les organisations professionnelles. Il convient donc de partir de ces débats avant de voir ensuite les prises de position des partenaires sociaux.

\section{Les débats au Conseil économique et social}

Les cinq avant-projets de loi soumis pour avis au Conseil économique et social ont connu des sorts divers ${ }^{20}$. L'avant-projet de loi relatif au règlement intérieur et au droit disciplinaire a fait l'objet de critiques de forme ou d'observations de caractère technique; le projet d'avis a été adopté par 54 voix (CGT-FO, CGC, CFTC, trois membres du groupe des entreprises nationalisées, groupe de l'artisanat, un membre du groupe de l'agriculture) contre 48 (CGT, groupe des entreprises privées, deux membres du groupe de

19 R. SOUBIE, «La négociation collective: chances et risques de la réforme en cours», Droit social, avril 1982, pp. 280-283; P. LANGLOIS, «La technique juridique et la réforme des conventions collectives, Droit social, avril 1982, pp. 284-287; R. SOUBIE, "L'obligation de négocier et sa sanction", Droit social, janvier 1983, pp. 55-62; A. SUPIOT, "Les syndicats et la négociation collective», Droit social, janvier 1983, pp. 63-73; G. BELLIER, «Le double niveau de négociation dans les lois Auroux: un atout pour la politique contractuelle», Droit social, janvier 1983, pp. 74-79 et surtout le numéro spécial de Droit social, novembre 1982: «La négociation dans l'entreprise».

20 Avis et rapports du Conseil économique et social, journal officiel, $n^{\circ} 6,14$ mars 1982. 
l'agriculture) et 51 abstentions (CFDT, groupes de la coopération, de l'agriculture, un membre du groupe des entreprises nationalisées). Dans son avis, «le Conseil économique et social rappelle aux Pouvoirs publics qu'il ne suffit pas de créer des droits pour les rendre effectifs. Encore faut-il prévoir les mesures fonctionnelles qui en permettront l'exercice. Ainsi, la mission permanente de contrôle de conformité confiée aux services de l'inspection du travail imposera nécessairement un important accroissement de ses moyens. De même, à défaut d'une amélioration sensible des conseils de prud'hommes, le recours à l'arbitrage de ces juridictions pour le contrôle de la proportionnalité de la sanction à la gravité de la faute entraînera des retards considérables et donc finalement une paralysie de ces instances».

Il en va un peu de même de l'avant-projet de loi relatif aux comités d'hygiène de sécurité et des conditions de travail. Après avoir reconnu que «s'il est une institution dont l'efficacité fait l'unanimité, c'est bien le comité d'hygiène et de sécurité», le Conseil économique et social fait un certain nombre de recommandations sur les missions du CHSCT, sa composition, son fonctionnement et s'attache en détail à la notion de danger imminent pour suggérer une procédure adéquate. Le projet d'avis a été adopté par 85 voix (CFDT, CGT, FO, CGC, CFTC, groupes de l'artisanat, des entreprises nationalisées, de la coopération, quatre membres du groupe de l'agriculture) contre 26 (CGT, huit membres du groupe des entreprises privées) et 45 absentions (UNAF, groupes de l'agriculture, des entreprises privées).

Concernant l'avant-projet de loi relatif au développement des institutions représentatives du personnel, le Conseil économique et social n'a pas été en mesure d'émettre un avis, le projet d'avis établi par la section par 49 voix contre 45 et 59 abstentions ayant été repoussé.

Pour le Conseil économique et social, «l'avant-projet de loi relatif à la négociation collective et au règlement des conflits du travail constitue, sinon la pièce maîtresse, du moins la clef de voûte de l'ensemble du dispositif législatif découlant du rapport remis par le ministre du travail au gouvernement en septembre 1981, dit «rapport Auroux». Cet avant-projet de texte a pour ambition d'atteindre l'un des quatre objectifs fixés dans le dit rapport: le renouveau de la négociation collective en vue de "concourir à assurer dans le pluralisme des opinions, la réussite d'une politique contractuelle active fondant le développement économique et social au service de l'emploi». Mais s'il partage l'objectif - faire de la politique contractuelle la pratique privilégiée du progrès social - les préoccupations gouvernementales mettre en oeuvre un droit qui stimule plus qu'un droit qui encadre - , si, compte tenu de l'importance du sujet, il s'attache à proposer pour nombre d'articles une nouvelle rédaction, modifiant des articles, en supprimant d'autres, le Conseil économique et social se démarque du projet Auroux sur 
deux points essentiels. S'il accepte l'obligation de négocier dans la branche, il estime «qu'il n'est pas actuellement souhaitable d'instituer une obligation de négociation dans l'entreprise». Par ailleurs, «le Conseil économique et social s'écarte sur un point important de l'avant-projet de loi, celui du droit d'opposition instauré par l'article L 132.24 au bénéfice d'une ou plusieurs organisations représentant dans une entreprise plus de la moitié des électeurs inscrits. Il considère, en effet, qu'une telle disposition constituerait une remise en cause de la capacité reconnue, jusqu'à présent, à chaque organisation représentative et une atteinte au pluralisme syndical, constante du syndicalisme français; il rejette en conséquence l'article ci-dessus indiqué et en demande la suppression». Le projet d'avis relatif à cette question a été adopté par 111 voix (CGT, FO, CGC, CFTC, groupes de l'agriculture, de l'artisanat, des entreprises nationalisées, des entreprises privées, de la coopération) contre 27 (CFDT, CGT) et 13 abstentions (UNAF et un membre du groupe de l'agriculture).

L'attitude adoptée à l'égard du dernier texte est, dans sa brièveté, encore plus caractéristique, traduisant une véritable fin de non-recevoir: «Le Conseil économique et social adhère à l'esprit du projet de loi, tel que l'exposé des motifs le précise, mais considère que l'expression des travailleurs passe aujourd'hui par différentes institutions existantes dans les entreprises: organisations syndicales, comités d'entreprise, délégués du personnel, comités d'hygiène et de sécurité, ainsi que par la hiérarchie fonctionnelle et opérationnelle. Le Conseil économique et social estime donc qu'au moment où le rôle et l'avenir de ces différentes structures sont modifiés ou en cours de modification, il est impossible d'envisager d'institutionnaliser de nouvelles forces d'expression des travailleurs.» Le projet d'avis a été adopté par 88 voix (CGT, FO, CGC, groupes de l'agriculture, des entreprises nationalisées, des entreprises privées, trois membres du groupe de la coopération), contre 45 (CFDT, CGT, CFTC, UNAF, un membre du groupe de l'agriculture, deux membres du groupe de la coopération) et 19 abstentions (groupe de l'artisanat, cinq membres du groupe de la coopération).

\section{Les positions du patronat}

Sans doute y a-t-il au sein du patronat des fractions qui peuvent considérer que les lois Auroux peuvent être à l'origine d'un véritable progrès social. Ainsi le Centre des jeunes dirigeants d'entreprise (CJD) estime que «le droit d'expression dans l'entreprise peut être une dynamique féconde, à la fois pour les salariés parce que c'est un des besoins fondamentaux des personnes humaines de s'exprimer, et pour les entreprises, car l'un des défis auxquels nous sommes confrontés dans les années 80 , c'est celui de l'innovation». De même, si le terme d'obligation peut être gênant, il n'en 
demeure pas moins que pour le CJD «la négociation c'est aussi un moyen de rapprocher les points de vue. En même temps, on donne de l'information. Ce peut donc être une façon d'aller vers ce fameux consensus si souvent souhaité et pour lequel trop peu de gens oeuvrent concrètement» ${ }^{21}$. Mais, d'une façon générale, il est possible de considérer que, dans son ensemble, le patronat manifeste une opposition idéologique profonde, est hostile à l'égard de quelques dispositions techniques, mais est désormais confronté à une véritable alternative stratégique.

L'opposition idéologique est sans fard, Yvon Chotard premier viceprésident du CNPF et responsable du secteur social estime que le rapport Auroux est une véritable opération démagogique: «la conception que Monsieur Auroux veut promouvoir repose sur une idée de démocratie économique et de citoyenneté d'entreprise, cette transposition du langage politique à l'entreprise n'a pas beaucoup de sens et c'est une source de profonde erreur. L'entreprise n'est pas une communauté politique... Dans ces conditions, parler de citoyenneté d'entreprise est un contre-sens. Parler de démocratie d'entreprise est un abus de langage. Non seulement la conception doctrinale est contestable, mais il se dégage du rapport Auroux, comme autrefois du rapport Sudreau, une vue manichéenne de l'entreprise. En effet, le rapport Auroux semble limiter l'entreprise à un face à face entre l'employeur et les syndicats» ${ }^{22}$. Même vision idéologique chez le représentant des PME: «À chacun sa définition de l'entreprise, écrit-il; en ce qui nous concerne ce n'est ni le lieu de l'exercice d'une nouvelle citoyenneté, ni un forum, ni le ring sur lequel s'affrontent les dirigeants d'un côté et les salariés de l'autre. L'entreprise, c'est essentiellement une communauté d'hommes et de femmes participant chacun, par son travail, à la réalisation d'objectifs destinés à satisfaire une clientèle; cet ensemble dynamique qu'est l'entreprise, soumis aux dures lois du marché, doit dégager des surplus qui assurent les besoins de la collectivité et qui permettent d'améliorer la protection sociale ainsi que le niveau et les conditions de vie» ${ }^{23}$.

Si on se place maintenant d'un point de vue technique, les positions du patronat peuvent être présentées comme suit. Concernant tout d'abord la lutte contre la précarisation de l'emploi et plus particulièrement la forme particulière que constitue le travail temporaire, les organisations patronales de ce domaine d'activité (UNETT, PROMAT, SPEMETT) estiment que les freins mis vont introduire des rigidités dans le fonctionnement du travail temporaire, génératrices de coûts, de chômage et qui dressent «des interdits

21 P. NOCTURNE, Le monde, 11 juin 1982.

22 Y. CHOTARD, «Le rapport Auroux», Droit social, avril 1982, p. 259.

23 J. BRUNET, «Le rapport Auroux: Le point de vue des PME», Droit social, avril 1982 , p. 267. 
beaucoup trop stricts dans un domaine où la souplesse est absolument nécessaire et reconnue par tous", point de vue repris par les autres organisations professionnelles du patronat. S'agissant des institutions représentatives et du pouvoir disciplinaire, les mesures adoptées sont estimées de nature à augmenter les charges ou à entraver les prises de décision. L'instauration d'un comité de groupe à partir de présomptions d'existence du groupe est une démarche inacceptable. N'est pas non plus acceptable le principe de l'obligation de négocier dans l'entreprise, le droit de véto des organisations syndicales; ces deux mécanismes risquent de bloquer la politique contractuelle qui a souvent été le fait d'organisations ouvrières minoritaires et de stériliser les négociations de branche. La mise en application obligatoire du droit d'expression à partir d'un seuil d'effectif bas et après un contrôle syndical sur les procédures comporte un risque très sérieux de détournement ou d'échec.

Les lois ayant maintenant été adoptées, se pose désormais pour le patronat la tactique à adopter à leur égard. Deux attitudes sont possibles ${ }^{24}$. La première, qu'on peut qualifier de réglementaire, consisterait à essayer de maintenir le plus possible la situation actuelle. La seconde, pouvait être dite dynamique, consisterait au contraire à transformer une contrainte en atout: la rencontre annuelle peut permettre de négocier une augmentation du temps de travail des machines et d'obtenir l'abandon de droits acquis au profit d'«acquis de substitution», retrouvant ainsi l'esprit des productivity bargainings inaugurés par ESSO à Fawley; l'expression directe peut constituer, intelligemment utilisée, un véritable contrepoids au renforcement du syndicalisme dans l'entreprise, encore faut-il pour cela transformer les cadres en animateurs - ce qui suppose décentralisation du pouvoir, information vivante et formation adaptée - et s'assurer de la maîtrise en revalorisant son rôle.

\section{Les positions syndicales}

Les organisations ouvrières reconnaissent que nombre de dispositions relevant des lois Auroux vont dans le sens de leurs revendications (par exemple en ce qui concerne le règlement intérieur, l'hygiène, la sécurité et les conditions de travail, la lutte contre le travail précaire, le renforcement des institutions représentatives du personnel en particulier en ce qui concerne les effectifs, les crédits d'heures, la formation ou l'exercice des droits, l'impulsion donnée à la négociation) même, si on peut éventuellement constater sur ces points quelques divergences de détail (par exemple, la CGC n'est pas op-

24 M. Millot, J.P. ROUlleAU, L'entreprise face aux lois Auroux, Editions d'organisation, 1983. 
posée par principe au travail temporaire; elle récuse le droit à une représentativité propre des organisations de cadres qui se fondent dans une centrale ouvrière; la CFTC préférerait le recours à la médiation obligatoire plutôt qu'à l'obligation de négociation). Mais c'est essentiellement le droit de veto et le droit d'expression qui introduisent des clivages au sein du monde ouvrier ${ }^{25}$. C'est qu'en effet, ainsi qu'on a pu à juste titre le relever «deux conceptions du syndicalisme sont, à cet égard, apparues dans le débat. L'une est plus sensible au rôle du syndicalisme dans la société. Dans cette conception, l'activité du syndicat en dehors de l'entreprise est finalement son activité essentielle. Cette activité est centrée sur le développement d'une politique contractuelle au sein des branches d'activité et sur un plan interprofessionnel... Dans l'entreprise, l'action revendicative passe essentiellement par les délégués. Cette conception correspond à une certaine vision de l'organisation sociale partagée, même s'il existe des divergences ponctuelles d'appréciation, par le CNPF et des organisations telles que FO, CFTC et CGC. Les tenants de la seconde conception considèrent que le syndicalisme trouve la source de sa légitimité dans l'organisation et l'action collective des travailleurs sur les lieux de travail. Ils sont plus attentifs à rechercher les moyens de permettre cette organisation et cette action, à partir d'une représentativité et d'une mobilisation faisant appel sur le terrain aux travailleurs eux-mêmes. Malgré leurs divergences sur le rôle respectif du syndicat et des travailleurs dans le changement social, la CFDT et la CGT se retrouvent dans ce second groupe ${ }^{26}$.

La CGT porte sur le dispositif Auroux un jugement globalement positif estimant qu'il rejoint pour une bonne part les préoccupations de la centrale ouvrière et va dans le sens du changement, notamment en ce qui concerne l'amélioration du système des conventions collectives et du droit de négociation, la limitation du pouvoir discrétionnaire des employeurs, le droit d'information des comités d'entreprise, l'amélioration de la situation des travailleurs soumis à la précarité de l'emploi. Mais elle relève un certain nombre d'insuffisances par exemple en matière de droit de grève, de droit de licenciement, et d'hésitations par exemple pour ce qui est du contrôle et de l'intervention des travailleurs sur les décisions et choix patronaux en matière économique, ou encore du caractère reconnu à la loi qui possède à ses yeux un important caractère unificateur; en matière de droit syndical, sans négliger les avancées enregistrées ce sont surtout les absences qui se font cruellement sentir: l'heure mensuelle d'information syndicale n'est pas

25 On trouvera, sous forme de tableau, une comparaison entre le droit positif ancien, les propositions Auroux et les revendications syndicales dans le document $n^{\circ} 125 / 81$ du 2 novembre 1981 de Liaisons sociales.

26 J.P. MURCIER, «La CFDT et les droits nouveaux pour les travailleurs», Droit social, juillet-août 1982, p. 536 . 
prévue, il n'y a pas de section syndicale inter-entreprise, de crédits d'heures pour le fonctionnement propre de l'organisation syndicale; manquent aussi les moyens indispensables à l'expression des salariés ${ }^{27}$.

La CFDT qui avait critiqué le rapport Auroux pour l'absence de dispositions concernant l'organisation du droit syndical dans les petites entreprises enregistre avec satisfaction que cette lacune a été comblée, regrette par contre la faiblesse des propositions nouvelles dans le domaine du droit syndical. Mais c'est surtout deux domaines essentiels qui constituent pour elle une percée significative dans le sens d'une démocratisation de la vie économique. L'obligation de négocier est l'une d'elle car «depuis 1968 la section syndicale, légalement reconnue, est restée pour une large part une coquille vide. Une fois respecté le cadre très général des conventions collectives, l'employeur est par exemple seul maître de la détermination des salaires réels ou de la répartition du temps de travail. Demain, partout, il lui faudra en débattre, à fond, une fois par an avec la section syndicale d'entreprise». Le droit d'expression des travailleurs en est une autre car il «a une ambition majeure: celle de libérer en même temps la parole et le savoir ouvriers pour changer le travail, son organisation et sa qualité et améliorer le produit du travail» ${ }^{28}$. Aussi à ceux qui sont sceptiques sur la portée de ce droit, trouvant que le texte qui le concerne a une allure «psy» et soixante huitarde, en ce qu'il "remplace la prise du pouvoir par la prise de parole» ${ }^{29}$, la CFDT réplique en y découvrant quatre finalités (amélioration des conditions de travail, amélioration de la qualité du travail, transformation de l'organisation du travail, modification des rapports hiérarchiques) et deux effets indirects (sur la productivité de l'entreprise et sur le syndicalisme lui-même, contraint de s'adapter pour tenir compte, positivement, des débats des groupes de travailleurs) ${ }^{30}$.

FO ne pouvait que souscrire à l'objectif du développement de la négociation collective notamment par le biais de la réunion annuelle dans les branches professionnelles pour discuter des salaires et de la réunion tous les cinq ans pour discuter des classifications. Par contre, trois orientations lui paraissent particulièrement dangereuses et parfois juridiquement contestables: l'obligation de négociation dans l'entreprise et la primauté de l'accord d'entreprise sur la convention de branche, cette dernière risquant, par là même, de se trouver neutralisée; l'arbitrage syndical en matière d'applica-

27 G. GAUME, «Le temps de la démocratie dans l'entreprise», Droit social, avril 1982, pp. 267-272.

28 E. MAIRE, Le Monde, 19 août 1982.

29 G. ADAM, «À propos du 'droit d'expression des salariés': réflexions critiques sur un texte sans importance», Droit social, avril 1982, pp. 288-290.

30 J.P. JACQUIER, «Droit d'expression: si c'était cela aussi la transformation d'une société?», Droit social, septembre-octobre 1983, pp. 561-563. 
tion des accords d'entreprise qui érige en juge «de ce qui est bon, moins bon ou seulement crédible en matière d'accord»; le droit de véto de deux organisations pour l'extension des conventions collectives qui conduirait une organisation syndicale ou patronale à s'opposer à l'exercice par le ministre de son pouvoir réglementaire. Quant au droit d'expression, FO ne pouvait que s'y montrer hostile, au nom d'une conception qui veut qu'il appartienne à la seule organisation syndicale «d'engager une responsibilité collective, de parler et d'agir au nom de la classe ouvrière» ${ }^{31}$.

Le point de vue de la CFTC est encore différent. Sa conception du syndicalisme, avec les notions d'humanisme et de bien commun qu'il implique, ne pouvait que la faire souscrire au droit d'expression des salariés - d'où son vote spécifique sur ce point au conseil économique et social. «Depuis 1875 , dans le cadre d'un rapport présenté à un congrès et tout au long des concertations qui ont abouti à la rédaction du rapport Sudreau, la CFTC a plaidé pour l'institution, dans les entreprises, de structures de dialogue décentralisées. Soutenant cette thèse, la CFTC n'exprime pas la moindre réserve quant à la capacité des organisations syndicales ou des institutions représentatives de travailleurs à prendre en charge les intérêts de ceux-ci. Elle considère simplement qu'il est normal, naturel, qu'au sein de chaque unité de travail, la plus décentralisée possible, le salarié ait la possibilité de s'exprimer sur les questions relatives à l'organisation et aux conditions de son travail» ${ }^{32}$. Par contre, comme FO, la CFTC est très réservée en ce qui concerne deux mesures susceptibles, selon elle, de remettre en cause la politique contractuelle: le seuil de représentation de $50 \%$ des organisations syndicales pour donner valeur à un texte et le droit de veto de deux organisations syndicales pour l'extension des conventions collectives.

\section{CONCLUSION}

Au delà des dispositions techniques qui ont retenu et retiendront encore largement l'attention des spécialistes du droit du travail et des relations professionnelles, les lois Auroux posent, comme on l'a vu, deux problèmes essentiels: celui du devenir de notre système de relations sociales, celui de la prise en charge par la collectivité de travail des aspirations des salariés dans toute leur diversité et leur évolution. En s'efforçant de stimuler la négociation au niveau des branches et de l'implanter au plan des entreprises, la loi tente de mettre en place un système de relations professionnelles qui encadre

31 P. HOFMAN, «Les «lois Auroux»: un droit qui encadrera ou un droit qui stimulera», Droit social, avril 1982, pp. 273-277. 565.

32 J. GRUAT, «Le point de vue de la CFTC», Droit social, septembre-octobre 1983, p. 
plus qu'il ne réglemente et qui fait une large confiance aux acteurs sociaux pour définir un état de droit. En mettant en place un dispositif original pour permettre aux travailleurs d'exprimer leur vécu quotidien et suggérer les moyens d'aménager conditions et organisation du travail, le législateur fait un pari sur les capacités d'initiative des travailleurs. Réunifier la collectivité de travail, permettre à chacun d'exercer ses responsabilités propres: tels semblent en définitive les repères de la cohérence du droit nouveau qui s'esquisse, fondé sur une conception claire et globale de la politique sociale ${ }^{33}$.

\section{$A$ «Common Sense Revolution» (Auroux)}

A «Common Sense Revolution»: that is the way Jean Auroux described the corpus of new rules made public in 1982. In order to understand the nature of this revolution - the changes it included and the reactions it provoked - the rationnel behind these new rules must be examined.

There are in fact three basic reasons for the changes. First, the Block-Lainé Commission (established by Mitterrand in May 1981) revealed the extent of social inequality, the failure of policies of participation, and the expansion of certain offensive management strategies, all of them affecting «individual work relations». The insufficiency of both representative institutions and the system of negotiation were also dealt with as problems affecting "collective work relations».

Secondly, a comparative study had indicated that many of the features of the proposed Auroux legislation were to be found in other European IR systems. Thirdly, the new legislation became logical in the light of the programme of the Left and the Socialist Manifesto.

The task facing the new Minister of Labour included putting together a logical programme and ensuring its application. The Auroux Report of September 1981 contained two fundamental concepts and four consequent series of measures. The first concept was that workers must be considered as fullfledged members of organizations. To accomplish this goal it would be necessary: to restore and enlarge workers' rights, to reconstitute the "work collectivity» by carefully regulating all exceptions to legislation (part-time and contractual work, work of limited duration, etc.). The second concept was that workers must be able to influence changes within organizations about decisions directly affecting them. The way to bring this about was to create a more meaningful role for representative institutions and reactivate an «active contractual policy».

33 J. AUROUX, «Un nouveau droit du travail?», Droit social, janvier 1983, pp. 2-4. 
As a result, four essential series of measures were adopted, involving:

- restoring and enlarging workers' rights by reforming internal regulations and disciplinary law, as well as asserting the right of expression within organizations (Law of August 4, 1982);

- reconstituting the "work collectivity» by clarifying the aims, and limiting the use, of temporary work («Ordonnance» of February 5, 1982), the contract of limited duration («Ordonnance» of February 5, 1982), and part-time work («Ordonnance» of March 26, 1982), as well as improving the rights of and protections for the workers concerned;

- creating a more significant role for union and employee representative institutions (Law of October 28, 1982), and integrating occupational health and safety committees in future with commissions determining working conditions (Law of December 23, 1982);

- relaunching the "contractual policy» with annual compulsory negotiation of salaries and duration of work (Law of November 13, 1982).

The debates of the Economic and Social Council revealed tensions threatening to produce ruptures in French society. Among them, the obligation to negotiate and the right of expression of workers within the organization particularly posed problems. Management clearly showed ideological opposition to the Auroux legislation, but found itself faced with an alternative: to do battle at the level of rules and regulations, or to turn the obstacles to its own advantage. If the CGT and CFDT, in spite of technical criticism, approved the new programme, the FO and CFTC were much more critical concerning the right of veto given to representative institutions and the right of expression.

\section{LA SYNDICALISATION DANS LE SECTEUR PRIVÉ AU QUÉBEC}

Préface: Gilles FERLAND - Introduction: Jacques BÉLANGER, Jean BOIVIN, Claude RONDEAU, Jean SEXTON - Le syndicalisme dans l'entreprise: tendances récentes et analyse, Claude RONDEAU et Jacques BÉLANGER - La syndicalisation dans le contexte économique québécois, Sidney INGERMAN - Commentaires: Bernard BONIN - L'impact du régime de relations du travail sur la syndicalisation, Gilles LAFLAMME - Commentaires: Hélène LEBEL - Les idées de réforme sur la syndicalisation au Québec depuis 1964, Jacques DESMARAIS - Table ronde: Organisation syndicale: difficultés et motifs de résistance, Pierre MERCILLE, Bertin NADEAU, Madeleine OLIVIER - Pourquoi et comment accroître la syndicalisation dans le secteur privé? Monique SIMARD, Raymond SLIGER - Commentaires: Gérard DION -L'entreprise peut-elle se passer d'un syndicat?, Charles PERREAULT - Commentaires: Fernand GAUTHIER, Paul-Martel ROY - La politique gouvernementale en matière de syndicalisation, Raynald FRÉCHETTE - Table sonde: Les réactions du milieu, Claire BONENFANT, Ghislain DUFOUR, Jean-Paul HÉTU - Syndicalisme, nouvelles technologies et incertitudes économiques, Guy CAIRE.

ISBN 2-7637-7030-4

1 volume, 276 pages - Prix $\$ 17.00$

Les Presses de l'Université Laval

Cité universitaire

C.P.2447, Québec, P.Q., Canada, GIK 7R4 